\title{
Misdiagnosis of Thoracolumbar Posterior Ligamentous Complex Injuries and Use of Radiographic Parameter Correlations to Improve Detection Accuracy
}

\author{
Frank Hartmann ${ }^{1}$, Thomas Nusselt ${ }^{1}$, Stefan Mattyasovszky ${ }^{2}$, \\ Gerrit Maier ${ }^{3}$, Pol Maria Rommens ${ }^{2}$, Erol Gercek ${ }^{1}$ \\ ${ }^{1}$ Center for Trauma and Orthopedic Surgery, Gemeinschaftsklinikum Mittelrhein, Ev. Stift Koblenz, Koblenz, Germany \\ ${ }^{2}$ Department of Orthopedics and Traumatology, University Medical Center of the Johannes Gutenberg University Mainz, Mainz, Germany \\ ${ }^{3}$ University Hospital of Orthopaedic Surgery, Pius-Hospital, Carl von Ossietzky University, Oldenburg, Germany
}

Study Design: Retrospective study.

Purpose: To evaluate radiological parameters as indicators for posterior ligamentous complex (PLC) injuries in the case of limited availability of magnetic resonance imaging.

Overview of Literature: Traumatic thoracolumbar spinal fractures with PLC injuries can be misdiagnosed on X-rays or computed tomography scans. This study aimed to retrospectively assess unrecognized PLC injuries and evaluate radiographic parameters as indicators of PLC injuries requiring surgery.

Methods: In total, 314 patients with type A and type B2 fractures who underwent surgical treatment between 2001 and 2010 were included. The frequency of misdiagnosis was reassessed, and radiographic parameters were evaluated and correlated.

Results: The average age of the patients was 51.8 years. There were 225 type A3/A4 and 89 type B2 fractures; 39 of the type B2 fractures $(43.8 \%)$ had been misdiagnosed as type A fractures. Type B fractures presented with a significantly higher kyphotic wedge angle and Cobb angle and a lower sagittal index (SI) than type A fractures. In addition, the normalized interspinous distance was higher in type B2 fractures. The significant mathematical indicators for PLC injuries were as follows: Cobb angle+kyphotic wedge angle $>29^{\circ}$; Cobb angle ${ }^{2}>170^{\circ}$; and vertebral angle/SI $>25$.

Conclusions: The results demonstrated that PLC injuries are frequently misdiagnosed. Correlations between certain radiological parameters associated with PLC injuries can be useful indicators of the presence of such injuries requiring surgery.

Keywords: Posterior ligamentous complex injury; Throacolumbar spinal fracture; Spine injury classification; Radiographic assessment

\section{Introduction}

A multicenter study conducted in Germany found that
AO type B distraction injuries of the spine represent $20.9 \%$ of all thoracolumbar spinal injuries $[1,2]$. The posttraumatic spinal instability due to these AO type B dis-

Received Dec 26, 2017; Revised Jul 19, 2018; Accepted Jul 20, 2018

Corresponding author: Frank Hartmann

Center for Trauma and Orthopedic Surgery, Gemeinschaftsklinikum Mittelrhein, Ev. Stift Koblenz, Johannes-Müller-Str 7, 56068 Koblenz, Germany

Tel: +49-261-1371306, Fax: +49-261-1371606, E-mail: hartmann.trauma@gmx-topmail.de 
traction injuries largely depends on the injury to the posterior ligamentous complex (PLC), which comprises the supraspinous ligament, interspinous ligament, ligamentum flavum, and facet joint capsules [3,4]. A PLC injury usually requires surgical intervention to prevent progressive and persistent deformity due to the loss of spinal tensile strength [4]; therefore, correct interpretation of the spinal stability is essential for appropriate treatment. The Magerl classification of thoracolumbar spinal fractures has certain limitations; therefore, to account for PLC integrity, Vaccaro et al. [5] introduced the thoracolumbar injury classification and severity score (TLICS). However, in the absence of typical radiological findings, such as an interspinous gap or a severe dislocation, interpretations of plain radiographs or computed tomography (CT) scans can lead to a high rate of misdiagnosis and the underestimation of fracture severity $[4,6]$. To focus on possible surgical interventions in the case of limited access to magnetic resonance imaging (MRI) and indeterminate ligamentous injuries, the AOSpine thoracolumbar spine injury classification system included a patient-specific modifier (M1) [2]. However, international spine surgeons have varied opinions with regard to the identification of PLC injuries in patients with presumed type A fractures [7].

This study aimed to retrospectively assess the frequency of unrecognized PLC injuries in our department and evaluate radiographic parameters and algorithms to improve the accuracy of PLC injury detection.

\section{Materials and Methods}

\section{Patients and methods}

We retrospectively reviewed data of 317 patients with thoracolumbar spinal fractures who underwent surgery in our department between 2001 and 2010. During that decade, MRI was not used to detect type B2 spinal injuries as extensively as it is now. Surgery was performed according to the recommendations of the German Society for Orthopaedics and Trauma (DGOU) in cases of type A3 and type A4 fractures (categorized according to the recent AOSpine thoracolumbar spine injury classification system [2]) with segmental kyphosis of more than $20^{\circ}$, significant spinal canal encroachment, or significant vertebral body destruction, in addition to type $\mathrm{B}$ and type $\mathrm{C}$ fractures [8]. Specifically, patients with type A3, type A4, and type B2 fractures were identified, who underwent open dorsal in- strumentation. (For reasons of simplification, type A3 and type A4 fractures will be referred to as type A fractures throughout the report.) Patients with type $\mathrm{C}$ fractures, tumors, or infections were excluded from the study. Therefore, the actual number of patients was 314 .

In part 1 of the study, we reassessed the documented pre- and intraoperative classifications of the fractures in the medical records in order to identify patients who were intraoperatively reclassified from type A to type B2 during open dorsal instrumentation and thereby determine the frequency of unrecognized PLC injuries.

In part 2 of the study, two experienced spine surgeons and one experienced radiologist used picture archiving and communication system-implemented measuring tools to classify the fractures and analyze the preoperative anteroposterior and lateral radiographs and CT scans with reconstruction images. We evaluated the established radiographic parameters [3,9]: (1) The kyphotic wedge angle was formed by lines drawn parallel to the upper and lower endplates of the fractured vertebra (Fig. 1); (2) the sagittal index (SI) was defined as the ratio of the anterior (a) and posterior (b) vertebral body heights $(\mathrm{SI}=\mathrm{a} / \mathrm{b})($ Fig. 1$)$; and (3) the modified Cobb angle of Daniaux was formed by lines drawn parallel to the upper endplate of the cephalad vertebra and a line drawn parallel to the lower endplate of the caudal vertebra (Fig. 2). A previous study described the interspinous distance (ID) as a radiographic sign of a PLC injury [5]. In this study, according to the report of

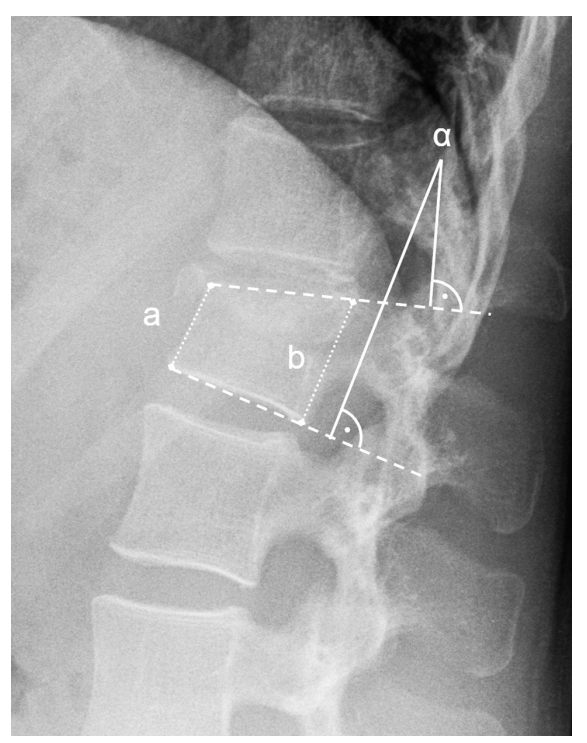

Fig. 1. Sagittal index: ratio between anterior (a) and posterior (b) vertebral body height (sagittal index=a/b). Kyphotic wedge angle $=\alpha$. 
Hiyama et al. [10], we evaluated the normalized ID as the distance of the spinous processes of the injured segment (s) normalized to the distance of one caudal segment (c) and expressed it as a percentage (ID [\%]=s/c) (Fig. 2).

Statistical analyses were conducted with IBM SPSS for Windows ver. 21.0 (IBM Corp., Armonk, NY, USA), Student $t$-test, and analysis of variance. All $p<0.05$ were considered statistically significant.

In part 3 of the study, we performed Pearson correlation analysis and evaluated combinations of radiographic parameters in mathematical formulas in order to increase the accuracy of PLC injury detection.

\section{Ethical approval}

All procedures performed in studies involving human

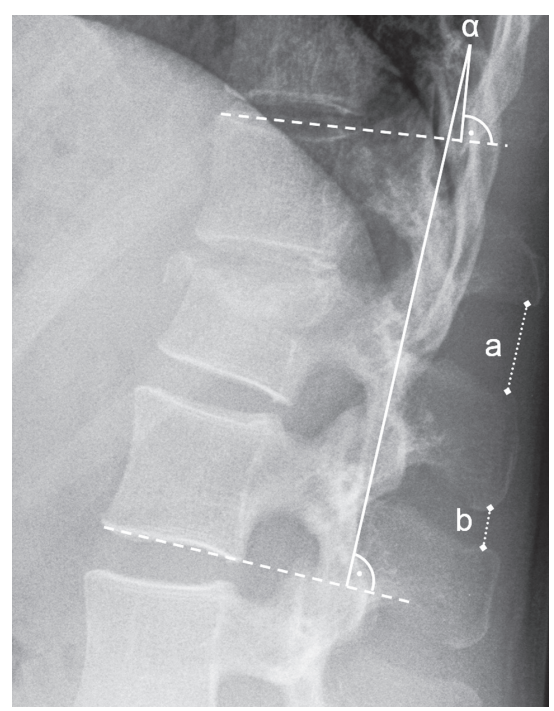

Fig. 2. Cobb angle $=\alpha$. Normalized ID: distance of the spinous process at the injured segment (a) normalized to the distance of one caudal segment (b) and expressed as a percentage (ID [\%]=a/b). ID, interspinous distance. participants were in accordance with the ethical standards of the institutional and/or national research committee and with the 1964 Helsinki declaration and its later amendments or comparable ethical standards. For this type of study, formal consent is not required. This study was approved from the Ethics Committee of the Landesaerztekammer of Rhineland-Palatinate, Mainz, Germany (approval no., 837.088.07 from 03 April 2007).

\section{Results}

In this study, a total of 317 patients underwent surgery for thoracolumbar spinal fractures between 2001 and 2010. The classification of the injuries according to the TLICS [5] revealed 225 type A fractures, 89 type B2 fractures, and 3 type $\mathrm{C}$ fractures (which were excluded from the study). The average age of the 176 male (56\%) and 138 female (44\%) patients was 51.8 years (range, $20-88$ years).

A review of the patients' pre- and postoperative medical records indicated that 39 of the 89 type B2 fractures (43.8\%) were initially misclassified as type A fractures and were not correctly identified until surgery. Radiological analysis revealed a significantly higher mean kyphotic wedge angle of $18^{\circ}$ (standard deviation [SD], $\pm 7.4^{\circ}$ ] for type $\mathrm{B} 2$ fractures relative to the corresponding kyphotic wedge angle of $11.1^{\circ}\left(\mathrm{SD}, \pm 6.0^{\circ}\right)$ for type A fractures (Fig. $3 \mathrm{~A})$. In addition, the mean Cobb angle was significantly higher for type $\mathrm{B} 2$ fractures $\left(16.85^{\circ} \pm 5.99^{\circ}\right)$ than for type $\mathrm{A}$ fractures $\left(10.38^{\circ} \pm 7.81^{\circ}\right)$ (Fig. $\left.3 \mathrm{~B}\right)$. Vertebral heights were significantly reduced to a mean SI of $0.63(\mathrm{SD}, \pm 0.24)$ for type $\mathrm{B} 2$ fractures compared to $0.73(\mathrm{SD}, \pm 0.14$ ) for type A fractures (Fig. 3C). The normalized ID was found to be $108 \%$ for type B2 fractures and 101\% for type A fractures; this difference was not significant.

In this study, Pearson correlation analysis revealed a significant positive correlation between kyphotic wedge
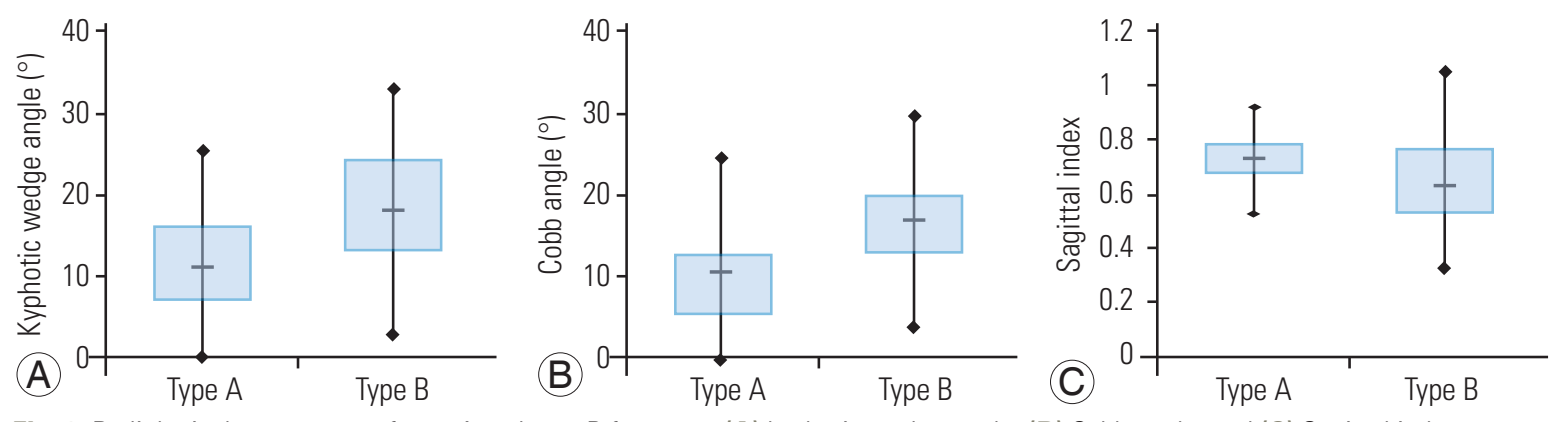

Fig. 3. Radiological parameters of type A and type B fractures: (A) kyphotic wedge angle, (B) Cobb angle, and (C) Sagittal index. 
Table 1. Odds ratios for mathematical combinations of radiological parameters for the prediction of posterior ligamentous complex injuries

\begin{tabular}{lc} 
Mathematical combination & Odds ratio \\
\hline Cobb angle+kyphotic wedge angle $\left(^{\circ}\right)$ & 6.9 \\
\hline Cobb angle+Cobb angle $\left(^{\circ}\right)$ & 2.3 \\
\hline Kyphotic wedge angle+kyphotic wedge angle $\left(^{\circ}\right)$ & 2.8 \\
Cobb angle ${ }^{2}\left({ }^{\circ}\right)$ & 3.5 \\
\hline Kyphotic wedge angle ${ }^{2}\left({ }^{\circ}\right)$ & 2.6 \\
\hline Kyphotic wedge angle/sagittal index & 4.5 \\
\hline Cobb angle/sagittal index & 2.2 \\
\hline
\end{tabular}

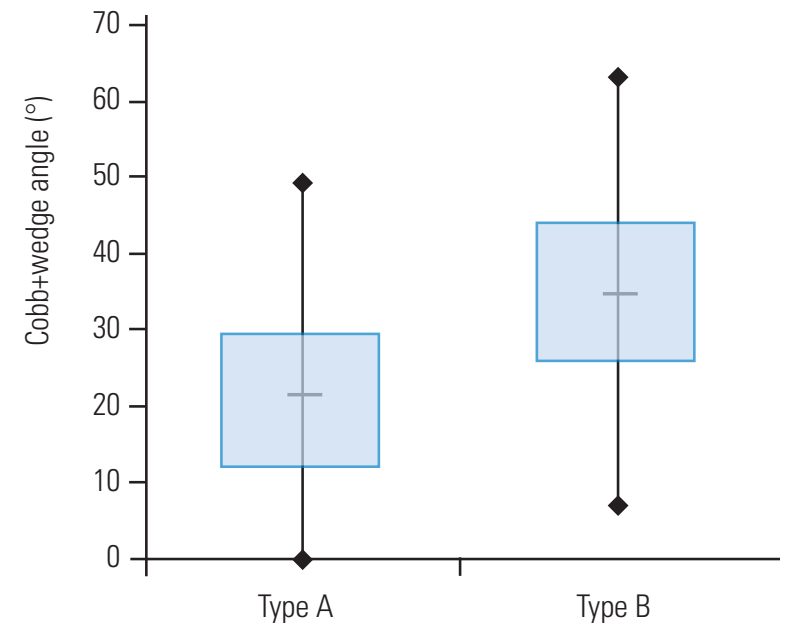

Fig. 4. Correlation of a radiological parameter of type $A$ and type $B$ fractures: Cobb angle+kyphotic wedge angle.

angle and Cobb angle in all types of fractures $(p<0.01)$. The SI showed a significant negative correlation with the kyphotic wedge and Cobb angles for both type A and type B2 fractures $(p<0.01)$.

The next step was to mathematically combine the parameters to find the highest odds ratios (ORs) for the prediction of PLC injuries (Table 1). First, we added up the parameters, and the OR was found to be 6.9 for the sum of Cobb and kyphotic wedge angles; analysis of fracture types showed that (Cobb angle+kyphotic wedge angle) $>29^{\circ}$ for $25 \%$ of type A fractures and $75 \%$ of type B2 fractures (Fig. 4). To give each parameter a higher weighting, next, we squared the parameters, and the OR was found to be was 3.5 for the square of the Cobb angle; analysis of fracture types showed Cobb angle ${ }^{2}>170^{\circ}$ for $25 \%$ of type A fractures and $75 \%$ of type B fractures (Fig. 5). For the SI, we performed more basic calculations until the

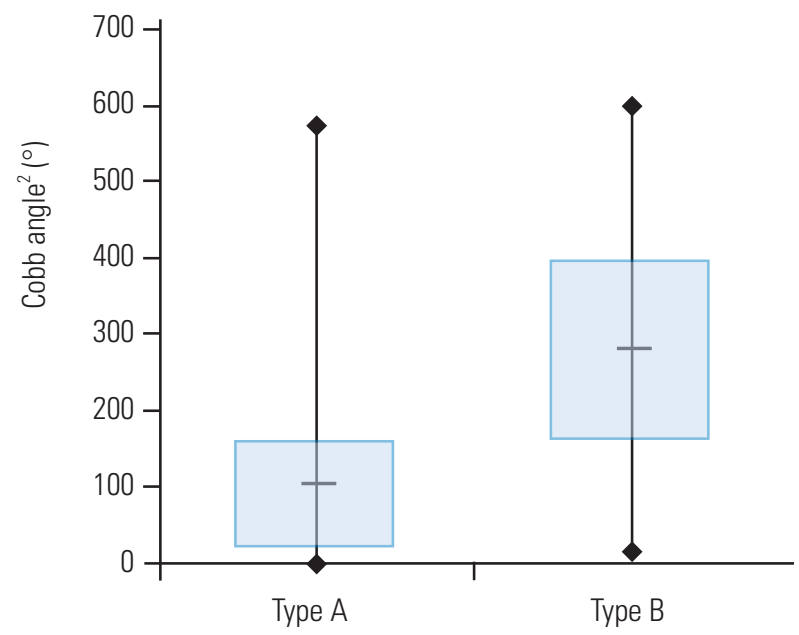

Fig. 5. Correlation of a radiological parameter of type A and type B fractures: Cobb angle ${ }^{2}$.

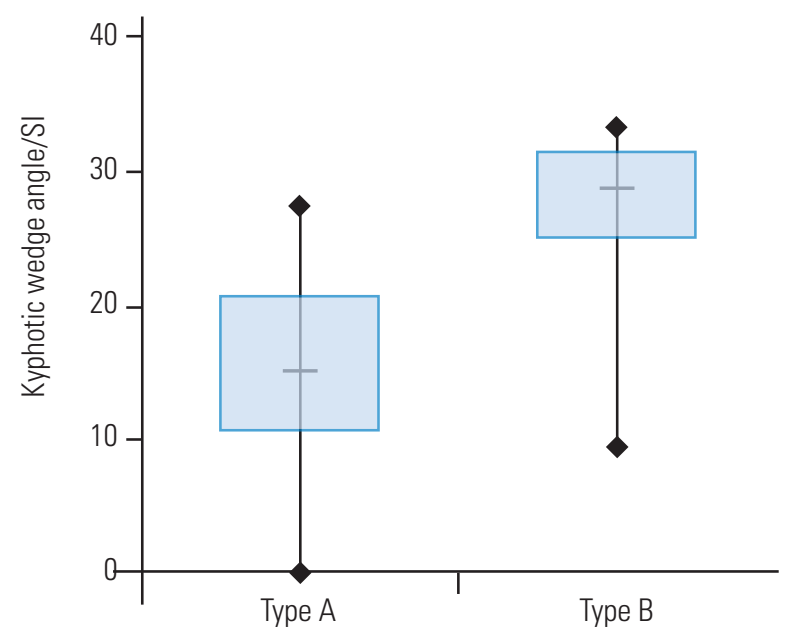

Fig. 6. Correlation of a radiological parameter of type $A$ and type $B$ fractures: kyphotic wedge angle/SI. SI, sagittal index.

kyphotic wedge angle divided by the SI showed the highest OR of 4.5; analysis of fracture types showed kyphotic wedge angle/SI $>25$ for $25 \%$ of type A fractures and $75 \%$ of type B2 fractures (Fig. 6).

\section{Discussion}

The various classification systems for thoracolumbar spinal fractures are intended to facilitate surgeons' treatment decisions by providing correct assessments of spinal instability. The TLICS uses an additional modifier to emphasize the key role of PLC integrity $[5,8]$. However, if the classification pathologies are identified on plain 
X-rays or CT scans, evaluating PLC integrity in primary discoligamentous injuries still remains difficult. Leferink et al. [6] retrospectively evaluated 160 patients with 49 type B fractures for whom plain X-rays and CT scans with two-dimensional reconstructions had been used as preoperative diagnostic tools. They found that approximately $30 \%$ of the type B fractures had been misdiagnosed preoperatively. Schnake et al. [11] observed that $41.9 \%$ of 93 type B injuries in a group of 361 patients were not recognized. These results are consistent with our misdiagnosis frequency of $43.8 \%$. In a retrospective study involving 65 patients and 85 vertebral fractures, Schröder et al. [12] demonstrated the advantage of using postprocessing algorithms in multidetector CT: the percentage of incorrectly classified type B fractures was reduced to $12.5 \%$.

On the basis of early biomechanical and clinical studies, a loss of vertebral body height of $>50 \%$, kyphosis of the thoracolumbar junction of $>20^{\circ}$, or increased ID implies a PLC injury $[4,10,13,14]$. For example, Schnake et al. [11] observed a reduction in the anterior vertebral body height and a segmental angle of $>15^{\circ}$ in $31 \%$ and $44 \%$ of type $B$ fractures, respectively; in $29 \%$ of the examined cases, typical PLC injury indicators were absent. Hiyama et al. [10] evaluated associations between radiological parameters in 40 thoracolumbar burst fracture cases involving potential PLC injuries that were diagnosed using MRI. The authors demonstrated that local kyphosis of $>20^{\circ}$ and increased ID correlated with PLC injury. Our results of a kyphotic wedge angle of $18^{\circ}$, a Cobb angle of $17^{\circ}$, and a reduction in vertebral body height to $63 \%$ were significantly correlated with PLC injury, whereas there was no significant difference in the ID between type A and type B fractures.

Part 3 of this study was distinct from prior investigations in that to increase the accuracy of potential PLC injury detection, radiological indicators were correlated and combined in mathematical terms. The results demonstrated that local kyphosis and a reduced vertebral body height appear to have a high predictive value. However, we could not evaluate a clear, simple formula for definitively identifying PLC injuries. To date, MRI is recommended for assessment of PLC injuries because of its high sensitivity $(79 \%-100 \%)[5,10]$, although reports of low specificity and low interobserver reliability suggest potential overdiagnosis and overtreatment of PLC injuries [10,15-17]. Additional disadvantages of MRI are its limited availability and high cost; according to the Organization for Economic Cooperation and Development,
$60 \%$ of MRI devices annually sold worldwide are bought in the United States and Japan [18]. As a result of these limitations regarding MRI, recent studies have evaluated ultrasound as a feasible tool for detecting PLC injuries and have reported achieving ultrasound sensitivity close to that of MRI $[19,20]$.

Our study had a few limitations. The sample size was small $(n=314)$, the number of observers was small $(n=3)$, and our focus was on operative cases alone, which could have biased evaluations because of the greater severity of such cases. Future follow-up investigations involving both non-operative and operative cases are required in order to evaluate parameters with the highest reproducibility and reliability and therefore improve precision and agreement among spine surgeons.

\section{Conclusions}

Correct diagnosis of PLC injuries is important for selecting the appropriate treatment of thoracolumbar spinal fractures, but these injuries are frequently unrecognized by using plain X-rays and CT scans. In the absence of MRI, spine surgeons should assess established radiological parameters, such as a high kyphotic wedge angle, a high Cobb angle, and a low SI. In addition, mathematical correlations of these parameters can also be a helpful tool to determine the requirement of surgical treatment in patients with thoracolumbar spinal fractures.

\section{Conflict of Interest}

No potential conflict of interest relevant to this article was reported.

\section{Acknowledgments}

The authors would like to thank the Institute of Medical Biostatistics, Epidemiology and Informatics of the University Medical Center of the Johannes Gutenberg University Mainz for their help with the statistical analysis of this study.

\section{References}

1. Reinhold M, Knop C, Beisse R, et al. Operative treatment of traumatic fractures of the thoracic and lumbar spinal column: part I: epidemiology. Un- 
fallchirurg 2009;112:33-42,44-5.

2. Vaccaro AR, Oner C, Kepler CK, et al. AOSpine thoracolumbar spine injury classification system: fracture description, neurological status, and key modifiers. Spine (Phila Pa 1976) 2013;38:2028-37.

3. Holdsworth F. Fractures, dislocations, and fracturedislocations of the spine. J Bone Joint Surg Am 1970;52:1534-51.

4. Magerl F, Aebi M, Gertzbein SD, Harms J, Nazarian S. A comprehensive classification of thoracic and lumbar injuries. Eur Spine J 1994;3:184-201.

5. Vaccaro AR, Lehman RA Jr, Hurlbert RJ, et al. A new classification of thoracolumbar injuries: the importance of injury morphology, the integrity of the posterior ligamentous complex, and neurologic status. Spine (Phila Pa 1976) 2005;30:2325-33.

6. Leferink VJ, Veldhuis EF, Zimmerman KW, ten Vergert EM, ten Duis HJ. Classificational problems in ligamentary distraction type vertebral fractures: 30\% of all B-type fractures are initially unrecognised. Eur Spine J 2002;11:246-50.

7. Schroeder GD, Kepler CK, Koerner JD, et al. A worldwide analysis of the reliability and perceived importance of an injury to the posterior ligamentous complex in AO type a fractures. Global Spine J 2015;5:378-82.

8. Verheyden AP, Holzl A, Ekkerlein H, et al. Recommendations for the treatment of thoracolumbar and lumbar spine injuries. Unfallchirurg 2011;114:9-16.

9. Keynan O, Fisher CG, Vaccaro A, et al. Radiographic measurement parameters in thoracolumbar fractures: a systematic review and consensus statement of the spine trauma study group. Spine (Phila Pa 1976) 2006;31:E156-65.

10. Hiyama A, Watanabe M, Katoh H, Sato M, Nagai T, Mochida J. Relationships between posterior ligamentous complex injury and radiographic parameters in patients with thoracolumbar burst fractures. Injury 2015;46:392-8.

11. Schnake KJ, von Scotti F, Haas NP, Kandziora F. Type $B$ injuries of the thoracolumbar spine: misinter- pretations of the integrity of the posterior ligament complex using radiologic diagnostics. Unfallchirurg 2008;111:977-84.

12. Schroder RJ, Albus M, Kandziora F, et al. Diagnostic value of three-dimensional reconstruction in CT of traumatic spinal fractures. Rofo 2003;175:1500-7.

13. Nagel DA, Koogle TA, Piziali RL, Perkash I. Stability of the upper lumbar spine following progressive disruptions and the application of individual internal and external fixation devices. J Bone Joint Surg Am 1981;63:62-70.

14. Vaccaro AR, Lee JY, Schweitzer KM Jr, et al. Assessment of injury to the posterior ligamentous complex in thoracolumbar spine trauma. Spine J 2006;6:524-8.

15. van Middendorp JJ, Patel AA, Schuetz M, Joaquim AF. The precision, accuracy and validity of detecting posterior ligamentous complex injuries of the thoracic and lumbar spine: a critical appraisal of the literature. Eur Spine J 2013;22:461-74.

16. Radcliff K, Su BW, Kepler CK, et al. Correlation of posterior ligamentous complex injury and neurological injury to loss of vertebral body height, kyphosis, and canal compromise. Spine (Phila Pa 1976) 2012;37:1142-50.

17. Rihn JA, Yang N, Fisher C, et al. Using magnetic resonance imaging to accurately assess injury to the posterior ligamentous complex of the spine: a prospective comparison of the surgeon and radiologist. J Neurosurg Spine 2010;12:391-6.

18. Organization for Economic Cooperation and Development. OECD health statistics. Paris: Organization for Economic Cooperation and Development; 2017.

19. Moon SH, Park MS, Suk KS, et al. Feasibility of ultrasound examination in posterior ligament complex injury of thoracolumbar spine fracture. Spine (Phila Pa 1976) 2002;27:2154-8.

20. Meinig H, Doffert J, Linz N, Konerding MA, Gercek E, Pitzen T. Sensitivity and specificity of ultrasound in spinal trauma in 29 consecutive patients. Eur Spine J 2015;24:864-70. 